\title{
Exploiting the complexity of the genome and transcriptome using pharmacogenomics towards personalized medicine
}

\author{
Reiner Hoppe ${ }^{1,2}$, Hiltrud Brauch ${ }^{1,2}$, Deanna L Kroetz ${ }^{3}$ and Manel Esteller, ${ }^{4 *}$
}

Abstract
A report of the 8th annual Pharmacogenomics and
Personalized Therapy meeting, Cold Spring Harbor
Laboratory, USA, 17-21 November 2010.

This meeting, a joint event of Cold Spring Harbor Laboratory (CSHL) and the Wellcome Trust and funded in part by the National Institute of General Medical Sciences (NIGMS), brings together scientists from many disciplines, from basic genomics to clinical medicine. They share a common interest in understanding the role of inherited variation in drug response phenotypes ranging from life threatening adverse drug reactions (ADRs) to lack of the desired therapeutic drug effect. Pharmacogenomics is a major component of the movement toward personalized or individualized medicine. This year, in addition to prominent examples of pharmacogenomic research, the meeting heard contributions on state-of-the-art next-generation sequencing, genome-wide analyses, genetic control of expression, epigenetics, and translational bioinformatics, complemented by presentations on ethical, legal, and societal issues. There were more than 130 international participants.

\section{Future scope of genomic medicine and key discoveries in pharmacogenomics}

The keynote lectures of the opening sessions were dedicated to the exploration of the future potential of pharmacogenomic applications in health care based on past and current developments. Eric D Green, Director of the National Human Genome Research Institute

\footnotetext{
*Correspondence: mesteller@idibell.cat

${ }^{5}$ Cancer Epigenetics and Biology Program (PEBC), Bellvitge Biomedical Research Institute (IDIBELL), Hospital Duran i Reynals, Av. Gran Via de L'Hospitalet 199-203, 08907 L'Hospitalet de Llobregat, Catalonia, Spain

Full list of author information is available at the end of the article
}

(NHGRI, Bethesda, USA), laid out the opportunities of the human genome sequence for genomic medicine (described as 'from base pairs to bedside'). The path of realization faces bottlenecks at many levels, but these can be overcome using techniques including functional genomic analysis (such as comparative genome sequencing and epigenetic landscaping), elucidation of human genomic variation (such as the HapMap Project and the 1000 Genomes Project), and identification of the genomic basis for rare monogenetic and common complex human disease (such as genome-wide association studies (GWASs) and post-GWAS approaches). Because the costs have become affordable, we can now perform routine whole-genome sequencing of individual human genomes. Yet the next-generation sequencing techniques will produce enormous amounts of sequence data that will require complex computational analyses and thorough interpretation of genomic information. Green presented a strategic plan of breaking down the tasks into five genomic domains, which will aid efforts to overcome current and future bottlenecks (Figure 1). For timely progress, this strategic plan should be revisited and refined every 5 years.

Pharmacogenetic markers and how they were discovered were presented by Michel Eichelbaum (Dr Margarete Fischer-Bosch-Institute of Clinical Pharmacology, Stuttgart, Germany). He highlighted prominent examples following a historic tour from the first pharmacogenetic trait at the beginning of the era of pharmacogenomics - the observation of the occurrence of hemolysis/death in relation to fava bean consumption in Mediterranean populations 3,000 years ago and its mechanistic explanation in modern times - to the discovery by Beutler, Waller and Löhr in 1956-7 of sensitivity to an antimalarial drug (primaquine) related to glucose 6-phosphate dehydrogenase deficiency. Some more recent examples are shown in Table 1. In the past 35 years, characterization of loss of function of drug metabolizing enzymes - including Eichelbaum's work on the cytochrome P450 (CYP) 2D6 poor metabolizer phenotype - has led to the understanding of the profound 


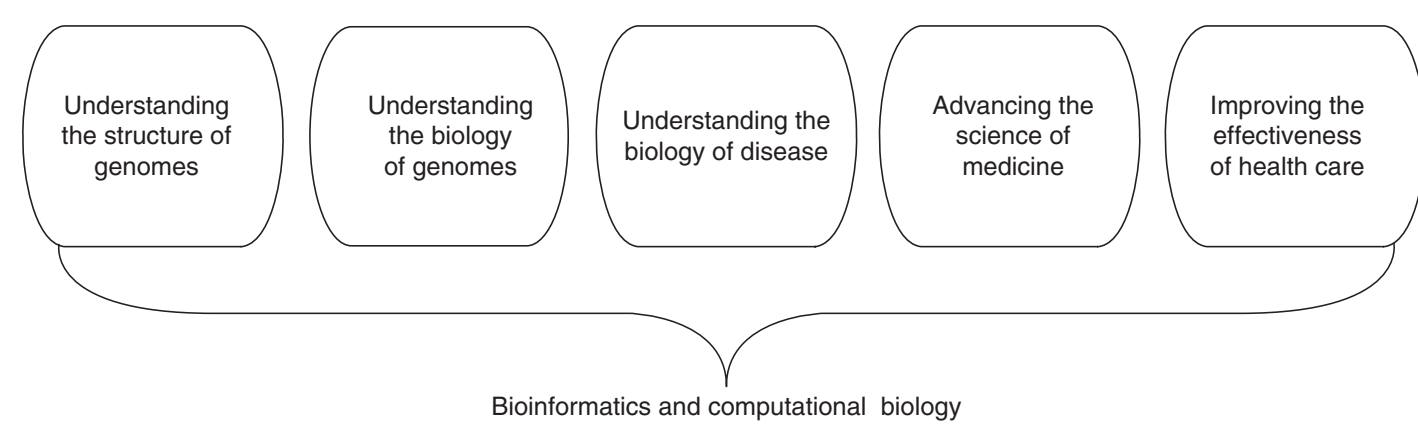

Education and training

1990-2003

Genomics and society

Human Genome Project

2004-2010

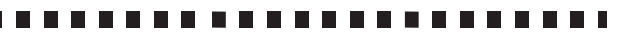

घ घ

$2011-2020$

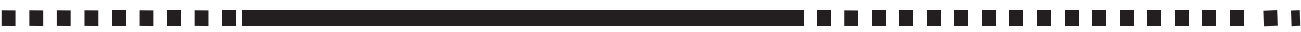

Beyond 2020

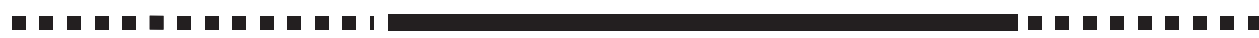

Figure 1. A strategic plan to achieve the goal of genomic medicine ('base pairs to bedside'). The scheme illustrates past and current accomplishments as well as future tasks in the five domains of genomic medicine, also embracing cross cutting elements: bioinformatics and computational biology, education and training, and genomics and society. Solid lines indicate major ongoing or expected activities and accomplishments across the domains at respective time intervals. Dashed lines indicate fewer activities, respectively.

Table 1. Examples of implementation of pharmacogenetics for the improvement of drug response and avoidance of adverse drug reactions

\begin{tabular}{|c|c|c|c|}
\hline Drug & $\begin{array}{l}\text { Marker and } \\
\text { genetic variants }\end{array}$ & Treatment application & Drug effect and risks \\
\hline Tamoxifen & $\begin{array}{l}\text { CYP2D6; PM alleles } \\
* 3,{ }^{*} 4,{ }^{*} 5,{ }^{*} 6 \text { and }{ }^{*} 7\end{array}$ & Breast cancer & $\begin{array}{l}\text { Prodrug that requires conversion into active metabolite } \\
\text { (endoxifen); PM patients have a higher risk of developing } \\
\text { recurrence than EM patients; CYP2D6 polymorphism explains } \\
38 \text { to 69\% of variable plasma endoxifen levels }\end{array}$ \\
\hline Warfarin & CYP2C9; VKORC1 & $\begin{array}{l}\text { Prevention of thrombo-embolic } \\
\text { events; maintenance dose is critical } \\
\text { (INR 2-3) }\end{array}$ & $\begin{array}{l}\text { Increased risk of thrombo-embolic events at INR }<2 \text { to } 3 \text {; } \\
\text { increased risk of bleeding at INR }>2 \text { to } 3 \text {; drug is the largest } \\
\text { cause of AEs and hospital emergency room visits }\end{array}$ \\
\hline Clopidogrel & $\mathrm{CYP} 2 \mathrm{C} 19^{*} 2$ & $\begin{array}{l}\text { Cardiovascular medicine to prevent } \\
\text { platelet aggregation }\end{array}$ & $\begin{array}{l}\text { Increased risk of cardiovascular event; explains 12\% of } \\
\text { variability }\end{array}$ \\
\hline Abacavir & HLA-B*5701 & AIDS & $\begin{array}{l}5 \% \text { of patients develop hypersensitivity (fever, skin rash, } \\
\text { gastro-intestinal symptoms, or eosinophilia); genotyping } \\
\text { before therapy reduces incidence of hypersensitivity and is } \\
\text { cost effective }\end{array}$ \\
\hline $\begin{array}{l}\text { Flucloxacillin, } \\
\text { amoxicillin-clavulanate }\end{array}$ & HLA-B*5701 & $\begin{array}{l}\text { Bacterial infections; } \beta \text {-lactam } \\
\text { antibiotics }\end{array}$ & $20 \%$ of DILI-related hospitalizations \\
\hline Simvastatin & OATP1B1*5 & $\begin{array}{l}\text { Control of elevated cholesterol } \\
\text { (hypercholesterolemia) }\end{array}$ & Increased risk of myopathy \\
\hline Cisplatin & TPMT, COMT & Chemotherapy & $\begin{array}{l}\text { Permanent, bilateral hearing loss (grade } 2-4 \text { ) in } 10 \text { to } 25 \% \text { of } \\
\text { adult patients and } 41 \text { to } 61 \% \text { of children }\end{array}$ \\
\hline
\end{tabular}

$\mathrm{AE}$, adverse event; AIDS, acquired immune deficiency syndrome; COMT, catechol-O-methyl transferase; CYP, cytochrome P450; DILI, drug-induced liver injury; EM, extensive metabolizer; HLA-B, human leukocyte antigen B; INR, international normalized ratio; OATP, organic anion transporting polypeptide; PM, poor metabolizer; TPMT, thiopurine S-methyltransferase; VKORC, vitamin K, epoxide reductase complex.

effects of inherited differences in drug metabolizing enzymes on the pharmacokinetics of a drug. Such differences can result in more than a 100 -fold difference in systemic drug exposure, with clinically important effects on drug response. Today, it is recognized that transporters, receptors, and genes of signaling pathways are 
also important pharmacogenomic components. It is now understood that genetic factors account for 20 to $95 \%$ of the variability in drug disposition (that is, absorption, distribution, metabolism and excretion) and effects; however, pharmacogenomic testing is not about a yes or no answer but about probabilities that a response/event occurs. Drug effects (response, resistance, and ADRs) cannot be explained by single genes or variants but are under the influence of multiple genetic and non-genetic factors (such as age, sex, body mass index and organ function). The identification of clinically useful markers therefore requires large pharmacogenomic studies with phenotypically well characterized study subjects, comprehensive allele coverage for the genetic description of these phenotypes, and consideration of confounders (such as drug adherence and co-medications).

\section{Innovations in genomic biology and implications for pharmacogenomics}

Deborah A Nickerson (University of Washington School of Medicine, Seattle, USA) highlighted the finding of new genetic variants and genetic signatures for diseases identified by exome sequencing. The complete set of exons (including splice sites and microRNAs) - referred to as the 'exome' - makes up only $1 \%$ of the human genome. By selecting the exome to sequence, important information about an individual can be obtained at a much lower cost than would be required for sequencing their entire genome. Assessment of the results of exome sequencing is based on knowledge of the genetic code and allows a more informative interpretation of genetic variants. Rare variants that typically provide a stronger indication of disease susceptibility can be detected. As a result of the sequencing of 1,000 exomes more than 360,000 novel variants have been identified, half of which change the resulting amino acid sequences. For example, examining CYP2C9 among 1,000 genomes revealed considerably more variants than those reported in public databases. Some of these result in amino acid changes at substrate binding sites, which means that exome sequencing could be highly relevant for pharmacogenomics discoveries.

The genetic control of drug response was addressed by David B Goldstein (Duke University, Durham, USA) using the example of variable response to drug treatment (pegylated interferon and ribavirin) of hepatitis C. He outlined discovery strategies for pharmacogenomic questions and reported on the determination of drug efficacy by analyzing sustained virological response rates. An association between sustained virological response and the variant rs12979860 of the interferon (IFN) $\lambda$-encoding gene $I L-28 B$ was identified by GWAS. There was inter-ethnic variation in the allele frequency of the protective reference allele (African Americans <
Hispanic < Caucasians < East Asians). Although no biological function is known, poor treatment responders have higher blood levels of expression of IFN-responsive genes. Likewise, GWASs identified an association between an ADR (severe anemia) and the chromosome $20 \mathrm{q} 13$ region. A functional interpretation is possible through a neighboring gene encoding an enzyme of ATP synthesis (ITPA), which contains two functional variants. Addressing the implications for diagnostic utility, drug development and evaluation, mechanistic insights, and control of sources of variability in clinical trials, Goldstein emphasized that although many drug responses are influenced by genetic variants, they cannot always directly be detected by GWASs but require further in-depth analysis.

The putative role of the transcriptome for pharmacogenomics was addressed by Thomas Gingeras (CSHL). He reported that eukaryotic transcriptomes are far from being understood because information stored in DNA sequences is complex, layered, and compartmentalized. Thus, the genome has to be re-annotated because of a growing number of newly identified transcripts, not only non-coding but also coding. He presented data from the Sanger Institute and ENCODE transcriptome projects based on deep sequencing and tiling array analyses of transcriptomes of various cell lines and tissues of multiple organisms. Subfractionation of cells enriches for certain RNA groups, resulting in the detection of new RNAs and RNA isoforms. The genome can be regarded as being much richer than currently thought because regions are interconnected and littered with start sites, with many functional elements on top of each other. Thus, a gene represents a 'coding/non-coding cluster in the genome', which serves as a template for up to eight transcripts. This refined view of unique transcriptomes should be applied to gaining a better understanding of gene regulation and its possible role in disease and drug response.

Copy number variants (CNVs) account for a major proportion of human genetic polymorphisms and they may have an important role in genetic susceptibility to human disease. Peter J Donnelly (Wellcome Trust Centre for Human Genetics, Oxford, UK) reported on a large genome-wide study of the Wellcome Trust Case Control Consortium (WTCCC) for the investigation of associations between $\mathrm{CNVs}$ and eight common diseases (16,000 patients - 2,000 with each of bipolar disorder, breast cancer, coronary artery disease, Crohn's disease, hypertension, rheumatoid arthritis, type 1 diabetes, and type 2 diabetes). They used an array targeting more than $11,700 \mathrm{CNVs}$, each detected by ten probes, and only 3,000 CNVs passed quality control. Although a small number of CNVs were found to be associated with Crohn's disease, type 2 diabetes, and breast cancer, these 
associations had been previously identified by tagging SNPs, from which it follows that CNVs cannot explain much of the missing heritability of common diseases. Moreover, analytical challenges in CNV studies include varying results attributable to different DNA sources (blood versus B cell lines) and observed high falsenegative rates from comparative $\mathrm{CNV}$ associations generated from SNP chips (Affymetrix versus Illumina platforms). Thus, $\mathrm{CNV}$ findings from case-control studies must be interpreted with care.

One of us (ME) explained the role of epigenetics in personalized medicine, particularly in oncology. He described that the contribution of DNA methylation and histone modifications, the two main epigenetic layers, to this area could be subdivided into epigenetic markers to predict drug sensitivity (pharmacoepigenetics) and genetic markers to predict response to epigenetic drugs (such as DNA demethylating agents and histone deacetylase inhibitors). The best example of pharmacogenetics is the prediction of good response to the chemotherapy drugs temozolomide and carmustine in gliomas in which there is silencing of the DNA repair enzyme $O^{6}$ methylguanine-DNA methyltransferase (MGMT) associated with promoter $\mathrm{CpG}$ island hypermethylation. Further examples included the epigenetic inactivation of the Werner gene and response to irinotecan in colorectal cancer. Recent results from his group have demonstrated that hypermethylation of the $B R C A 1$ breast cancer gene CpG island is a marker of good response to poly ADPribose polymerase (PARP) inhibitors in breast cancer cells. This finding might significantly increase the number of breast cancer patients that could benefit from these therapies outside the high-risk inherited forms of the disease. He also showed that the genes involved in the epigenetic machinery, such as DNA methyltransferases and histone modifiers, can also undergo genetic changes related to differential sensitivity to epigenetic drugs. Examples of genetic amplification, deletion, and mutation of these genes are described in the literature, and a frameshift mutation in the histone deacetylase 2 (HDAC2) gene is associated with resistance to histone deacetylase inhibitors. Emerging research in the genomics and epigenomics arena will provide us with new epigenetic candidates for the personalized treatment of human malignancies.

\section{The role of bioinformatics in pharmacogenomics}

Steven E Brenner (University of California, Berkeley, USA) showed the long-term exponential growth of sequence data and its recent disruptive increase in growth. His experience of RNA splice regulation using RNA sequencing technology showed that the majority of alternative splicing events contain previously unannotated exonic structures; he extrapolated from this to highlight the great potential of using the vast amounts of sequence data in the emerging field of pharmacotranscriptomics. The talk introduced a community experiment to evaluate computational methods for phenotype prediction based on genomic variation (the Critical Assessment of Genome Interpretation, CAGI: http://genomeinterpretation.org), modeled on the Critical Assessment of Structure Prediction (CASP) exercise for protein structure prediction. There is an urgent need to interpret the multitude of variations in expression, allele-specific expression, splicing, transcript structure, and common and rare exonic variants. Dozens of prediction algorithms exist for this purpose, but their utility is not well understood. The CAGI experiment presented a number of datasets for the prediction of phenotypic consequences, including a pharmacogenomic dataset of over 50 breast cancer cell lines exposed to over 50 different drugs that will be relevant for the prediction of drug response. Within the personal genome project the genomes of ten individuals are being investigated for the prediction of 50 health- and disease-related phenotypes. CAGI ultimately had over 100 prediction submissions from seven countries. CAGI aims to understand the diversity of mechanisms of genome variation, identify bottlenecks in genome interpretation, inform critical areas of future research, and connect researchers from diverse disciplines whose expertise is essential to methods for genome interpretation.

\section{Ethical, economical and societal aspects of pharmacogenomics}

The cost-effectiveness of pharmacogenomics with respect to challenges and opportunities in an era of decreasing test costs was addressed by David L Veenstra (University of Washington, Seattle, USA). A stated lack of evidence of clinical utility is a frequent reason for health insurers to refuse to reimburse patients for pharmacogenomic testing. To better assess such evidence, the Evaluation of Genomic Applications in Practice and Prevention (EGAPP) initiative is developing a systematic process to capture the validity and utility of emerging genetic tests for clinical practice. A novel approach, comparative effectiveness research, aims to ensure that much more useful data will be collected (as compared with standard clinical trials) and better methods developed for understanding the differences in drug effectiveness among different patient groups, such as risk-benefit modeling. Lastly, the value of conducting additional research should be weighed against the cost of conducting such research using quantitative value of research analyses.

\section{Future trends in pharmacogenomics}

Integrated presentations from pharmacologists and human genome biologists highlighted the incredible 
opportunities for pharmacogenomic applications in optimizing drug therapy. Rapid advances in genomic technologies are being quickly adopted by pharmacogenomics researchers and will fuel progress in this area. The overarching need for the collection of large numbers of well phenotyped drug-treated populations was a central theme of most presentations, as were the difficulties associated with identifying appropriate replication cohorts. Future advances in human genomics and their application to pharmacogenomics are expected to bring continued advances to this important area of translational medicine.

\section{Competing interests}

The authors declare that they have no competing interests.

\section{Author details}

'Dr Margarete Fischer-Bosch-Institute of Clinical Pharmacology, Auerbachstrasse 112, 70376 Stuttgart, Germany. ${ }^{2}$ University of Tuebingen, Geschwister-Scholl-Platz, 72074 Tuebingen, Germany. ${ }^{3}$ Department of Bioengineering and Therapeutic Sciences, University of California, 1550 Fourth Street, Box 2911, Bldg 19B-584E, San Francisco, CA 94143-2911, USA. ${ }^{4}$ Institució Catalana de Recerca i Estudis Avançats (ICREA), Barcelona, Catalonia, Spain. ${ }^{5}$ Cancer Epigenetics and Biology Program (PEBC), Bellvitge Biomedical Research Institute (IDIBELL), Hospital Duran i Reynals, Av. Gran Via de L'Hospitalet 199-203, 08907 L'Hospitalet de Llobregat, Catalonia, Spain.

Published: 17 January 2011

doi:10.1186/gb-2011-12-1-301

Cite this article as: Hoppe R, et al.: Exploiting the complexity of the genome and transcriptome using pharmacogenomics towards personalized medicine. Genome Biology 2011, 12:301. 